Citation Network Analysis for supporting Continuous Improvement in Higher Education

Claudia Colicchia

University of Hull, Hull University Business School, Logistics Institute

Cottingham Road, Hull, UK, HU6 7RX

e-mail: c.colicchia@hull.ac.uk; Tel.+44 (0)1482 347550

Alessandro Creazza (corresponding author)

University of Hull, Hull University Business School, Logistics Institute

Cottingham Road, Hull, UK, HU6 7RX

e-mail: a.creazza@hull.ac.uk; Tel.+44 (0)1482 347586

Fernanda Strozzi

LIUC University, School of Industrial Engineering

Corso Matteotti 22, 21053, Castellanza, Italy

e-mail: f.strozzi@liuc.it; Tel. +39 0331572364 


\title{
Citation Network Analysis for supporting Continuous Improvement in Higher Education
}

\begin{abstract}
Continuous improvement in Higher Education can be supported by effective literature reviews to unveil contemporary and current educational needs and lay the foundations of programmes of study. As no discipline remains static, the aim of this paper is to present a methodology for conducting literature reviews that can complement traditional content-based reviews by revealing the dynamic evolution of a discipline. This methodology is represented by Citation Network Analysis (CNA), a collection of tools that help to detect the dynamics of a field through computer-based systematic analyses of its bibliographic data. Notwithstanding its potential, CNA has been seldom adopted to conduct literature reviews. In this paper, CNA was applied to the evolving field of Logistics and Supply Chain Management education. Results provide evidence of the benefits of CNA for the identification of key issues, trends, and evolutionary trajectories supporting continuous improvement in Higher Education in a more scientific and objective way.
\end{abstract}

\section{Keywords}

Citation Network Analysis, Literature Review, Higher Education, Continuous Improvement, Logistics and Supply Chain Management 


\section{Introduction}

Requirements of modern Higher Education challenge academics at both national and global levels to devise market and research driven programmes of study, as these contribute significantly to shaping the future direction of many professions and industries. Hence, the updating of skills and knowledge for students becomes imperative (Perera et al. 2016; Lutz and Birou 2013). It is recognized that this process of continuous improvement can be supported by effective reviews of the literature to unveil the contemporary and current educational needs and lay the foundations of programmes of study in Higher Education (Gravier and Farris 2008).

As no discipline remains static (Wang and Bowers 2016), the aim of this paper is to present a methodology for conducting literature reviews able to reveal the dynamic evolution of a field of study and to embrace the limitless expansion of knowledge. The adopted approach is based on Citation Network Analysis (CNA), a collection of tools that help to detect the dynamics of a field through computer-based systematic analyses of its bibliographic data. Even if the adoption of different measures based on citation data is recently gaining popularity to assess authors' or journals' influence (Budd and Magnuson 2010), techniques of network analysis have been seldom applied to conduct literature reviews, also within the area of Higher Education research (Calma and Davies 2015). The successful adoption of such an approach to other contexts, e.g. Kim et al. (2016), Kajikawa et al. (2007), proves its potential value in the identification of trends, evolutionary trajectories and key issues that are influencing the development of knowledge within a field in a more scientific and objective way compared to traditional reviews.

As a relevant application field in Higher Education, we adopt the above mentioned approach to Logistics and Supply Chain Management (L\&SCM) education. The study of L\&SCM is essential to understand how the effective and efficient management of logistical activities and supply chains can help businesses in fulfilling customer expectations while making profit (Christopher 2010; Gattorna 2006). In a world of constant transformation, talented logistics and supply chain management professionals are essential to master the complexity and acceleration of nowadays global networks of companies and to create and support competitive advantage for businesses (Wong et al. 2014). Offering a provision of L\&SCM programmes in Higher Education able to reflect such complexity and turbulent evolution emerges as a priority for universities across the globe. Hence, the field of L\&SCM education has recently gained considerable attention and this has caused a growth in the number of related publications. As a consequence, literature reviews are necessary to support the continuous improvement in the development of Higher Education programmes of study in L\&SCM by depicting the state of 
the art and identifying trends and trajectories that may influence the future direction of the field. However, to the best of authors' knowledge, only Gravier and Farris (2008) provide a literature review of the field. This gap is more apparent when it comes to the evolutionary aspect of this field. In fact, the authors adopt a traditional descriptive methodology for reviewing the literature and their perspective is static. Additionally, this study happens in a timely fashion, given that existing studies predict a shortage of a workforce of L\&SCM professionals equipped with market relevant and research driven skills to be developed through appropriate programmes of study (Lutz and Birou 2013).

As the challenge for Higher Education institutions to offer relevant and up-to-date programmes of study in a process of continuous improvement is common to any field, we believe that our study and the adopted methodology can support academics in succeeding in this.

The subsequent sections present the adopted methodology and its application to the field under study. These will be followed by a discussion of the obtained results and the strengths and weaknesses of the adopted methodology. Final remarks conclude the paper.

\section{The adopted methodology: Citation Network Analysis}

CNA can help to visualize and to extract information from papers based on their citations (Colicchia and Strozzi 2012). In fact, the underlying assumption of CNA is that fields of research are not meaningless set of papers in terms of citations. A citation network can be depicted as a directed graph and it represents a field of research as a network, where the nodes are the papers and the links are the citations among them. The links are directional ties represented by arrows and the direction of the arrows illustrates the flow of knowledge. A link from article A to article B means that article B cites article A since it built on the knowledge and information contained in article A to develop its own contribution. Assuming that researchers tend to cite each other to position their work in the field to create new knowledge, the analysis of this network can shed light on knowledge transformation and creation in a specific scientific field (De Nooy, Mrvar and Batagelj 2011; Hummon and Doreian 1989).

\section{Data collection and software packages for data processing and analysis}

The starting point of the literature review based on CNA is the collection of data, which needs to be carried out applying a search string (composed of combinations of keywords) to a citation 
database (e.g. the Web of Science-Core Collection database, WoS). Collected data allow building and visualizing the citation network.

Four, freely-available, software packages are proposed in this paper to process and analyse the data extracted from citation databases. Histcite (Lucio-Arias and Leydesdorff 2008) is a system designed to help organise and analyse the papers retrieved in topical searches and it converts the retrieved information into a ".net" file. This file extension is necessary for conducting analyses through CNA tools. The Science of Science (Sci2 2009) homepage defines the software package as "a modular toolset specifically designed for the study of science. It supports the temporal, geospatial, topical and network analysis and visualization of datasets". The Pajek software package (De Nooy, Mrvar and Batagelj 2011) is used for the analysis and visualization of large networks. The Visualization of Similarities (VOS) viewer (Van Eck and Waltman 2009) is a freely-available computer program for creating, visualizing and exploring bibliometric networks of science.

\section{Data Analysis}

Citation data can be analysed in the first instance through a series of basic statistics available in the HistCite software package, i.e. distribution of published scientific articles in the considered time window, distribution of citations in the considered time window, most frequently cited articles, countries with the highest number of citation network articles.

Besides basic statistics, the following analyses are proposed:

- Main Path Analysis

- Burst Detection

- Authors' keywords co-occurrence network analysis

- VOS clustering and mapping

\section{Main Path Analysis}

The Main Path Analysis was specifically designed to identify few papers in a citation network that are able to describe the evolution of the field (Hummon and Doreian 1989). This does not mean that the algorithm is able to identify the "best" papers or the most originals ones. It detects the path of citations more often walked in the full citation network, to reach the most recent papers starting from the oldest ones. Taking into account the chronological citations' network among the identified papers on the Main Path it is possible to describe the dynamic evolution of the research field under investigation, making its transformation over time visible (Hummon 
and Doreian 1989). In fact the Main Path highlights the articles that are based on prior articles to transform or create new knowledge in the field and act as a reference point for more recent works (Lucio-Arias and Leydesdorff 2008).

The steps to perform Main Path Analysis through the Pajek software package are the following:

1. Quantifying the traversal weight of the citation, i.e. the extent to which a citation is necessary to connect articles. Pajek offers three different methods: Search Path Count - it takes into account all paths from each source article (i.e. not citing any others) to each sink article (i.e. not cited by others). The traversal weight is given by the ratio between the number of paths including the citation and the overall number of paths; Search Path Link Count - it considers all paths from all vertices to the sink; Search Path Node Pair - with this method each vertex is regarded as a source and a sink.

2. Extracting the main path component. Using the computed traversal weights the main path component that will identify the main streams of the considered literature can be extracted. A cut-off value between 0 and 1 needs to be selected to remove all connections with a lower value, to extract the most important connected component.

For more details, interested readers can refer to de De Nooy, Mrvar and Batagelj (2011).

\section{Burst Detection}

The published literature in a particular research field can be seen as a sequence of topics that appear, grow in intensity for a period of time, and then fade away. The appearance of a topic in a document stream is signalled by a "burst of activity. Kleinberg (2002) developed a formal approach for identifying such "bursts". The Burst Detection algorithm (Kleinberg 2002) aims at identifying the increase in the frequency of use of certain words by authors and is available in the software package Sci2. The title words or the authors' keywords (referred to as Original Keywords) are considered to provide a fairly detailed focus of an article and the analysis of their evolution over time has proven to be a useful method for identifying research trends (Fu, Wang and Ho 2013). The algorithm generates a list of the word bursts, together with the time intervals in which these bursts occurred. Because the algorithm is case-sensitive, it is advisable to normalize the keywords before applying it. The process of normalization separates the text into token words, normalizes them in lowercase, removes the "s" at the end of words, removes dots (.) from acronyms, deletes the stop words, and applies the English Snowball stemmer (http://snowball.tartarus.org/algorithms/english/stemmer.html). 


\section{Authors' keywords co-occurrence network analysis}

The topical analysis of the field can be conducted through the study of the authors' keywords co-occurrence network that can be derived from the citation data through the software package Sci2. The nodes are the authors' keywords and the link weights represent how many times the words appear together in the same papers. Co-occurrence analysis is based on the assumption that the paper author keywords constitute an adequate description of its content or of the links that the paper established among concepts. The presence of many co-occurrences around the same word(s) may correspond to a research theme. Co-occurrence analysis reveals patterns and trends in a specific discipline by measuring the association strengths of terms representative of relevant publications produced in a discipline (Ding, Chowdhury and Foo 2001). Word cooccurrence networks are weighted and undirected. Also in this case it is advisable to normalize the keywords before creating the co-occurrence network, by applying the same procedure described in the previous section.

\section{VOS clustering and mapping}

The co-occurrence keyword network can be also analysed using VOS viewer software package that implements and visualizes the VOS clustering technique. VOS mapping determines the locations of items in a map by minimizing a function depending on a similarity measure, ASij, between items defined as:

$$
A S_{i j}=\frac{c_{i j}}{c_{i} c_{j}}
$$

where cij is the measure of the co-occurrence of keywords $i$ and $j$ in the same document and ci and cj are the expected number of co-occurrences of $i$ and $j$ under the assumption that the cooccurrences of $i$ and $j$ are statistically independent (Van Eck and Waltman 2009). VOS viewer software package can be applied to the keywords co-occurrence network after normalization.

\section{Application of the methodology to the field of L\&SCM education}

\section{Data collection}

The bibliographic research was performed using WoS, which is a scholarly database that contains "over 55 million records from the top journals, conference proceedings, and books in 
the sciences, social sciences, and arts and humanities to find the high quality research most relevant to your area of interest"' (Web of Science 2014).

The literature search was performed in January 2016 using WoS with the following string: (("logistics education" OR "supply chain") AND education AND (skills OR capabilities OR competences))

The search was performed using "Topic" as a field (which includes Title, Abstract and Keywords) and selecting all the databases included in WoS (Science Citation Index Expanded SCI-EXPANDED, Social Sciences Citation Index SSCI, Arts \& Humanities Citation Index A\&HCI, Conference Proceedings Citation Index-Science CPCI-S, Conference Proceedings Citation Index-Social Science \& Humanities CPCI-SSH, and Emerging Sources Citation Index ESCI). The search was performed setting "All years" in the Timespan field.

Since the word "logistics" is widely used in different contexts, it was combined with the word "education" to avoid results not related to the field under study. Furthermore, the words "skills", "capabilities" and "competences" were included in the search string with the aim to select papers focused on skills to be delivered to students through programmes of study. In fact, literature shows that these words are often used interchangeably. Furthermore it is acknowledged that they represent the foundation of programmes of study (Barrie 2007), that should be built on the development of an appropriate set of generic skills (Jackson 2016), also including more specialist ones on specific disciplines (Sohal 2013).

A network consisting of 66 nodes was constructed (Figure 1). It comprises one main connected component of 13 nodes (Figure 2) and some isolated nodes.

XXXXXXXXXXXXXXXXXXXXXXXXXXXXXXXXXXXXXXXXXXXXXXXXXXXX

Figure 1. Citation Network (66 nodes)

XXXXXXXXXXXXXXXXXXXXXXXXXXXXXXXXXXXXXXXXXXXXXXXXXXXX

XXXXXXXXXXXXXXXXXXXXXXXXXXXXXXXXXXXXXXXXXXXXXXXXXXXX

Figure 2. Main Connected Component (13 nodes)

XXXXXXXXXXXXXXXXXXXXXXXXXXXXXXXXXXXXXXXXXXXXXXXXXXXX

Basic statistics

Figure 3a) presents the distribution of the published papers included in the citation network and Figure $3 b$ ) shows the distribution of citations received by these articles. For the analyses we used HistCite software package version 12.03.17. 
The greatest number of articles was published in 2013 (25 articles), when a special issue on L\&SCM education was published on Supply Chain Management: an International Journal. Overall, there has been a substantial increase of articles published over the last years and a concurrent increase of the number of citations received by these articles, confirming the growing interest in this field.

\section{XXXXXXXXXXXXXXXXXXXXXXXXXXXXXXXXXXXXXXXXXXXXXXXXXXXX}

Figure $3 \mathrm{a} / 3 \mathrm{~b}$. Distribution of published scientific articles and citations XXXXXXXXXXXXXXXXXXXXXXXXXXXXXXXXXXXXXXXXXXXXXXXXXXXX

Table 1 presents the ranking of papers in terms of number of citations received, where the local citation score (LCS) refers to the count of citations to a paper within the data set of retrieved papers for this study and the global citation score (GCS) shows the total number of citations to a paper from other articles in the WoS. This table provides a first indication of the relevant papers in the field that have contributed to the development of knowledge, acting as a reference for later works. This list includes papers focused on providing a state of the art of logistics education with the aim to support the development of appropriate frameworks to support the design of logistics programmes (i.e. Lutz and Birou 2013; Lambrechts et al. 2012; Gravier and Farris 2008). Interestingly, within the most frequently cited articles, great attention is paid to the alignment of programmes of study with the requirements of future employers (see for example Sodhi, Son and Tang 2008; Murphy and Poist 2007). In fact, even though universities need to balance the needs of their customers (i.e. students, their parents and their employers) that can be initially different, it seems that eventually the outcome of an employable and market relevant L\&SCM professional is the key priority for all three (Murphy and Poist 2007).

XXXXXXXXXXXXXXXXXXXXXXXXXXXXXXXXXXXXXXXXXXXXXXXXXXXX

Table 1. Most frequently cited ten articles XXXXXXXXXXXXXXXXXXXXXXXXXXXXXXXXXXXXXXXXXXXXXXXXXXXX

Table 2 presents the list of countries with the highest number of articles. The top three countries, i.e. USA, UK and Australia, highlight the criticality of designing L\&SCM programmes, especially for those English-speaking countries able to "sell" their offered educational provision globally. 
XXXXXXXXXXXXXXXXXXXXXXXXXXXXXXXXXXXXXXXXXXXXXXXXXXXX

Table 2. Countries with the highest number of citation network articles

XXXXXXXXXXXXXXXXXXXXXXXXXXXXXXXXXXXXXXXXXXXXXXXXXXXX

\section{Main Path Analysis}

The Main Path of the articles citation network is shown in Figure 4 and it comprises 5 articles, which are labelled with the first author's surname and publication year. To obtain this main path the method chosen in this study to quantify the citation traversal weight is the Search Path Count because it counts all paths between each source and sink. The other methods (see Data Analysis section) are only recommended when specific node(s) are more important, which does not apply to this study. To extract the main path component, we used the default value of Pajek software package (version 64 2.05), i.e. 0.5.

\section{XXXXXXXXXXXXXXXXXXXXXXXXXXXXXXXXXXXXXXXXXXXXXXXXXXXX}

Figure 4. Main Path Component

XXXXXXXXXXXXXXXXXXXXXXXXXXXXXXXXXXXXXXXXXXXXXXXXXXXX

Reading the Main Path papers it is possible to identify the key issues in the area of L\&SCM education and to see an evolution of the key trends affecting the design of curricula in the field. The two "source" papers, i.e. Murphy and Poist (2007) and Gravier and Farris (2008), highlight the importance of soft skills related to business and management, which are highly valued by employers. According to these authors "logisticians should be managers first and logisticians second" (Murphy and Poist 2007, 423). This necessity is related to the major change the logistics discipline has undergone since the early 1990s, transforming itself from a "standalone" discipline strictly related to transportation and logistics to a part of supply chain management related to the integration of business processes within a firm and between interdependent organisations. Gravier and Farris (2008) also confirm the importance of a process of continuous improvement of L\&SCM programmes, that need to be constantly redesigned in response to a dynamic behaviour of a continuously evolving sector.

More recently, Lutz and Birou (2013) provide a state of the art of logistics education. Their findings, based on a survey of logistics syllabi, provide an analysis of the offered educational provision in the area of L\&SCM, in terms of learning objectives, content and adopted teaching styles. While providing this state of the art, the authors discuss some trends in the discipline. According to their study, universities should adopt an integrative approach to the development 
of skills, to be able to offer market relevant programmes of study. These skills should include general and specific knowledge, social, decision-making, problem-solving and time management skills. In terms of content, new current topics such as supply chain sustainability need to be included to provide students with updated knowledge on what is happening in the field. Finally, the importance of partnerships and collaboration with local companies is highlighted by the authors to support students' learning process.

After Lutz and Birou (2013) the Main Path bifurcates with Hohenstein, Feisel and Hartmann (2014) and Jirsak and Krsnakova (2015). These two articles reflect the newest trends in the area of L\&SCM. With the increasing globalization of supply chain networks, human resource management in supply chain management emerges as a top priority for firms and it is the focus of the work published by Hohenstein, Feisel and Hartmann (2014). According to these authors, besides a set of content-specific and managerial skills, it is important to provide students with a global mindset to be able to effectively manage global processes and compete in the dynamic and globally oriented market environment. On the other hand, Jirsak and Krsnakova (2015) discuss the importance of providing students with the latest knowledge in the field to support their success on the job market. Consistently with the trends in the discipline, according to these authors it is essential to transfer to students the required competences for managing supply chain sustainability and the knowledge on how to effectively use big data in the supply chain and master the complexity and interconnectivity of modern supply networks.

\section{Burst Detection Algorithm}

In Figure 5 and Figure 6 the results of the application of the Burst Detection Algorithm are shown (obtained using the software package Sci2 version 1.1 beta). The algorithm was applied respectively to Title words (Figure 5) and Original Keywords (Figure 6) after normalization. In the horizontal bar graph visualisation the $\mathrm{x}$-axis represents time, while the horizontal bars correspond to records in the dataset, i.e. normalized words.

XXXXXXXXXXXXXXXXXXXXXXXXXXXXXXXXXXXXXXXXXXXXXXXXXXXX

Figure 5. Burst detection of title words XXXXXXXXXXXXXXXXXXXXXXXXXXXXXXXXXXXXXXXXXXXXXXXXXXXX

Figure 6. Burst detection of original keywords 
Looking at the evolution of the words in titles (Figure 6), it is interesting to note that the word "logist" burst into scene starting from 1993, while the word "chain" appeared later on in 2010, thus confirming the evolution of the field and the related change in skills requirements as above explained. The graph highlights a burst of the words "skill", "level", "innov", "qualiti", "improv" between 2005 and 2008. Also in this case, the key issues arising from the main path are confirmed by this algorithm. During these years the field shows a greater and more formalised interest towards the definition of the required skills for L\&SCM graduates at different levels. The focus is on the continuous improvement of the quality of the offered educational provision. The introduction of appropriate innovation and the redesign of the curricula are deemed as critical to ensure the achievement of this quality. The time frame of this evolution, overlapping with the main path, is consistent with the first widespread and well consolidated endeavours globally to introduce formalised programmes of study in the specific field of L\&SCM. Finally, a burst of the word "sustain" is detected in 2010. The increase in the use of this word in titles proves the great relevance of the topic of supply chain sustainability, and the importance of consider it in the design of L\&SCM programmes.

Looking at the original keywords (Figure 7), the words "suppli" and "chain" burst into scene in 2003, when the discipline of supply chain management started to be widely developed. In fact it was necessary to achieve a complete conceptualisation of the discipline to have the burst of the same words in the titles of the papers, which occurred in 2010 (see above). The key issues and their evolutionary pattern described above are confirmed in the original keywords graph: the words "innov" and "quality" appeared in 2007-2008, while a burst of the word "sustain" is again detected in 2010.

\section{Authors' keywords co-occurrence network analysis}

Figure 7 is the authors' keywords co-occurrence network. The layout of this network is obtained applying the Kamada-Kawai algorithm through the use of the Pajek software package (Kamada and Kawai 1988). This algorithm is force-directed and aims to reduce the edge crossing in the representation of a network. Taking into account the weights of the links, it is able to visualise the nodes that are connected by stronger links closer to each other and thus this visualization may help to detect correlated nodes. Thanks to this layout, different "clusters" of authors' keywords can be recognised, corresponding to key issues discussed in the literature. 
XXXXXXXXXXXXXXXXXXXXXXXXXXXXXXXXXXXXXXXXXXXXXXXXXXXX

Figure 7. Original keywords network (size of arcs proportional to their weight) XXXXXXXXXXXXXXXXXXXXXXXXXXXXXXXXXXXXXXXXXXXXXXXXXXXX

Cluster A includes keywords related to the key focus of this literature review. The words "logist", "suppli", "chain", "manag", "skill", "educ" are present at the heart of this network, coherently with the used search string to collect data. Interestingly, the abovementioned words are tightly related to "talent" and "mindset". In fact according to the literature it appears that the ultimate aim of logistics curricula should be the development of talented professionals in the area. The key issue is that there will be a shortage of qualified L\&SCM professionals in the coming years (Ellinger and Ellinger 2014; Hohenstein, Feisel and Hartmann 2014; Lutz and Birou 2013). Hence, one of the most common themes that dominated the L\&SCM education field is the development of programmes of study seeking to prepare future logistics and supply chain professionals. Also, as highlighted in the Main Path, the importance to transfer to students the right mindset to be able to manage modern supply chains is confirmed by this analysis. A global mindset is defined as a "way of being" that, building upon a set of management skills, provides supply chain professionals with the right experiences and perceptions to be able to define global supply chain strategies for increasingly global organisations (Harvey et al. 2013). Thus it will be a central requirement for logisticians and supply chain managers and its development will be one of the key challenges in designing programmes of study in the field (Hohenstein, Feisel and Hartmann 2014).

Cluster B includes keywords (i.e. "teach" and "learn") related to the teaching and learning strategy. This cluster refers to the body of knowledge focused on L\&SCM teaching methods. Many contributions appeared in the literature with the aim to analyse methods for generating interest in the discipline among students and to transfer to them the right skills and abilities (see Gravier and Farris 2008). Among them, the literature discuss as key competences those related to leadership, communication, interaction in multicultural environment, relationships management and team building capabilities (Mangan and Christopher 2005) - as highlighted by the keywords "interact", "leadership", "interpers", "relationship" in the graph. Consistently with the main path analysis, recent literature highlight that the skills related to management can contribute to work performance of logistics and supply chain professionals more than those of logistics nature (Thai and Yeo 2015). Some authors discuss the relevance of innovative and interactive teaching methods to deliver those skills. "Active" learning (Soshko et al. 2005), game-based learning (Sedelnikova and Emelyanova 2014) and consultancy-based learning 
(Bak and Boulocher-Passet 2013) are discussed for their potential to provide students with experience at solving real life problems, increase their motivation and engagement and develop business-related competences. Other authors addressed the theme of providing work experience by exploiting the interactions with industry, in terms of industry guest speakers and internships (Sohal 2013).

Cluster $\mathrm{C}$ refers to the importance of training for the employability of L\&SCM graduates. In the graph the strong links among the words "train", "human" and "resource" reflect the debate present in the literature on the correlation between the success of L\&SCM graduates and the level of received training (Menon 2012; Popa, Tanasescu and Radulescu 2009). These words are strictly related to the word "job". Employability and marketability of L\&SCM graduates is becoming more and more important (Wong et al. 2014). The key research question has evolved over time from "What should a logistics and supply chain management programme look like?" to "Is the current provision meeting industry's needs?". Hence the presence in the literature of many contributions focused on understanding employers' perspectives on the desirable skills of recent graduates (Espinoza 2015; Jirsak and Krsnakova 2015; Wu et al. 2013). However, since a mismatch among course offering and the needs of industry in L\&SCM area is still generally perceived (Wong et al. 2014), further investigations are needed to investigate current and future industry's needs. While the literature offers the results of surveys to investigate employers' perspective in different contexts (e.g. Gammelgaard and Larson 2001; Murphy and Poist 1994; Wong et al. 2014; Sohal 2013), in-depth insights still need to be provided (Onar et al. 2013). Also, the students' perspective is generally overlooked in the literature (Rahman and Qing 2014).

Finally, authors' keywords included in cluster D (i.e. "big", "data", "inform", "commun", "analyt") highlight the importance of one of the key trends in the discipline of L\&SCM, as highlighted by the Main Path Analysis. While data science, predictive analytics, and big data are becoming increasingly relevant to supply chain management, the literature recognises that related skills and knowledge are crucial to develop future supply chain leaders and discusses how these can be implemented in the curriculum (Schoenherr and Speier-Pero 2015; Waller and Fawcett 2013).

\section{VOS clustering and mapping}

The VOS mapping algorithm was applied to authors' keywords co-occurrence network with the VOS software package version 1.6.3 (Figure 8). Overall the figure presents three main groups of keywords. Looking at the keywords included in these groups, it is possible to say 
that the group at the bottom-right corner is related to the learning and teaching strategy, while the one at the bottom-left corner is focused on curriculum design in terms of content and skills. Between them the mapping algorithm shows a third cluster labelled with the word "innov", confirming the importance of a process of continuous innovation in curriculum design which should embrace both the topics/skills included in the programmes of study and the adopted learning and teaching strategy to deliver the content and transfer the skills. The defined boundaries of these groups of keywords with no overlaps among them according to this algorithm suggest that the keywords included in the different groups are not often used together. This may imply that the study of the relationships among innovation in curriculum design, learning and teaching strategy, and topics and skills to be included in a programme of study in the area of L\&SCM is an unexplored research area.

XXXXXXXXXXXXXXXXXXXXXXXXXXXXXXXXXXXXXXXXXXXXXXXXXXXX

Figure 8. Authors' keywords VOS mapping

XXXXXXXXXXXXXXXXXXXXXXXXXXXXXXXXXXXXXXXXXXXXXXXXXXXX

\section{Discussions}

The results of the Main Path Analysis, the Burst Detection algorithm, Authors' keywords cooccurrence network analysis, and of the VOS clustering and mapping have yielded some insights into the main issues, evolutionary key trends and implications for research and practice in the field of application.

An emerging original finding is represented by the relevance of the interconnection among innovation in curriculum design, learning and teaching strategy, and topics and skills to be included in a programme of study. According to the knowledge flow generation and evolution, these areas have received a considerable but disparate attention in the literature on L\&SCM education. The analyses have specifically highlighted the need for bringing together the separate efforts towards an integrated approach to skills development and content design, learning and teaching strategies for knowledge transfer, through continuous innovation and improvement of programmes of study. As an overall result, such an integrated approach should be able to equip the L\&SCM professionals of tomorrow with that global mindset, i.e. an "integrative" combination of skills/capabilities/competences and awareness of new and evolving trends, which is recognised by the literature as the key challenge for high graduates' employability in a global and multicultural environment. This is deemed to pave the way for 
continuous improvement in the development of attractive, market relevant and current programmes of study.

Additionally, the adopted methodology allowed identifying the most influential papers in the extant literature and in the generation of knowledge over time, allowing researchers to focus directly and in the first instance on the scientific contributions that have shaped the advancements of the field and have been referred to in the development of researches.

The results of our research complement the findings of traditional content-based reviews and provide benefits for the development of the field of L\&SCM education, including:

- a groundwork for future research through the acknowledgement of the most influential past scientific contributions;

- a clear representation of the flow of knowledge generation over time, allowing for clear identification of the evolution of the field of L\&SCM education consistently with the evolution of L\&SCM as a discipline;

- a quantitative literature-based detection of emerging topics that are under-represented and require additional investigation, and of the linkages among topics;

- an analysis of the field that does not rely on subjective criteria for selecting papers and on pre-defined coding schemes to classify research contributions, but that relies instead on objective measures and algorithms;

- an assistance for identifying areas that align with the evolving educational goals of L\&SCM;

The output of this study permits to discuss the potential of the adopted methodology. First of all, it is a blind methodology that offers an original perspective in the analysis of a field of research. This is empowered by the use of computer-based systematic analyses and software packages that through algorithms are able to process data and information, for a prompt tracking of the status of a field. Through the analysis of the citation network it is possible to have objective measures of highly cross-referenced influential papers in a field and to capture through different tools and analyses the intellectual structure and evolution of the field under study, its main areas of research and promising directions for future research. In terms of originality of the methodology, this innovative combination of network analysis tools also delivers a fresher approach to complement the traditional content-based reviews, as illustrated by the presented application to the field of L\&SCM. 
This methodology has also some limitations. The main criticism is that taking into account the citations only it may not be completely informative about the real contribution of a paper to the body of knowledge (Shema 2013). Another criticism is that citation data are retrieved from databases, such as WoS, that, although quite comprehensive, include only a fraction of scientific publications. A last issue often discussed is the so-called "Matthew effect", i.e. the rich get richer. This means that researchers tend to cite papers of well-known researchers that already received a high number of citations, because they tend to regard these papers as reliable sources of information due to their reputation and popularity.

\section{Conclusions}

This paper presents a methodology for conducting literature reviews based on CNA and proposes an original set of analyses on bibliographic data. This methodology, despite its acknowledged potential in other fields, has rarely been applied so far, also in the area of Higher Education. We then applied this methodology to the field of L\&SCM education.

This study contributes to the current body of knowledge on L\&SCM education by analysing the evolution of this field of research, trends and emerging topics that are under-represented and require additional investigation. Moreover, from a broader perspective this paper provides an additional contribution: the described application to the field of L\&SCM education showcased the distinctive features of the methodology that could be of benefit for application to other fields. Through it researchers could gain a sense of the full picture of a field, or benefit from the decomposition of the citation network in its main components. This can help academics in further developing the body of knowledge of a field and to support the development of programmes of study from a practical perspective through the identification of the key issues, emerging trends and evolutionary trajectories.

Despite the discussed limitations, from a general viewpoint the interesting output of this study is not merely the visualisation of the citation network through colourful charts, but how CNA can be exploited as a research tool to support dynamic analyses for continuous improvement in Higher Education.

\section{References}

- Bak, O., and V. Boulocher-Passet. 2013. "Connecting industry and supply chain management education: exploring challenges faced in a SCM consultancy module." Supply Chain Management: an International Journal 18 (4): 468-479. 
- Barrie, S. C. 2007. "A conceptual framework for the teaching and learning of generic graduate attributes." Studies in Higher Education 32 (4): 439-458.

- Budd, J. M., and L. Magnuson. 2010. "Higher Education Literature Revisiting: Citation Patterns Examined." Research in Higher Education 51 (3): 294-304.

- Calma, A., and M. Davies. 2015. "Studies in Higher Education 1976-2013: a retrospective using citation network analysis." Studies in Higher Education 40 (1): 421.

- Christopher, M. 2010. Logistics and Supply Chain Management. $4^{\text {th }}$ Ed. FT Prentice Hall

- Colicchia, C., and F. Strozzi. 2012. "Supply chain risk management: a new methodology for a systematic literature review." Supply Chain Management: An International Journal 17 (4): 403-418.

- De Nooy, W., A. Mrvar, and V. Batagelj. 2011. Exploratory Social Network Analysis with Pajek. Cambridge University Press. Cambridge.

- Ding, Y., G. G. Chowdhury, and S. Foo. 2001. "Bibliometric cartography of information retrieval research by using co-word analysis." Information Processing \& Management 37 (6): 817-842.

- Ellinger, A. E., and A. D. Ellinger. 2014. "Leveraging human resource development expertise to improve supply chain managers' skills and competencies." European Journal of Training and Development 38 (1/2): 118-135.

- Espinoza, O. 2015. "Postsecondary educational needs of the wood products industry in the us northern great lakes region." Wood and Fiber Science 47 (1): 56-73.

- Fu, H., M. Wang, and Y. Ho. 2013. "Mapping of drinking water research: A bibliometric analysis of research output during 1992-2011." Science of the Total Environment 443: 757-765.

- Gammelgaard, B., and P.D. Larson. 2001. "Logistics skills and competencies for supply chain management." Journal of Business Logistics 22 (2): 27-50.

- Gattorna, J. 2006. Living supply chains: how to mobilize the enterprise around delivering what your customer want. FT Prentice Hall

- Gravier, M. J., and M.T. Farris. 2008. "An analysis of logistics pedagogical literature Past and future trends in curriculum, content, and pedagogy." International Journal of Logistics Management 19 (2): 233-253. 
- Harvey, M. G., R. Fisher, R. McPhail, and M. Moeller. 2013. “Aligning global organizations' human capital needs and global supply-chain strategies." Asia Pacific Journal of Human Resources. 51 (1): pp. 4-21.

- Hohenstein, N.O., E. Feisel, and E. Hartmann. 2014. "Human resource management issues in supply chain management research A systematic literature review from 1998 to 2014." International Journal of Physical Distribution \& Logistics Management 44 (6): 434-463.

- Hummon, N.P., and P. Doreian. 1989. "Connectivity in a citation network: the development of DNA theory." Social Networks 11 (1): 39-63.

- Jackson, D. 2016. "Skill mastery and the formation of graduate identity in Bachelor graduates: evidence from Australia." Studies in Higher Education 41 (7): 1313-1332.

- Jirsak, P., and L. Krsnakova. 2015. "Adherence of logistics education process to current business requirements." Efficiency and Responsibility in Education. 212-222.

- Kajikawa, Y., J. Ohno, Y. Takeda, K. Matsushima, and H. Komiyama. 2007. "Creating an academic landscape of sustainability science: an analysis of the citation network." Sustainability Science 2: 221-231.

- Kamada, T., and S. Kawai. 1998. “An Algorithm for Drawing General Undirected Graphs." Information Processing Letters 31: 7-15.

- Kim, S., C. Colicchia, and D. Menachof. 2016. "Ethical Sourcing: An Analysis of the Literature and Implications for Future Research." Journal of Business Ethics doi:10.1007/s10551-016-3266-8.

- Kleinberg, J. 2002. "Bursty and Hierarchical Structure in Streams." Proceedings 8th ACM SIGKDD International Conference on Knowledge Discovery and Data Mining.

- Lambrechts, F., T. Taillieu, S. Grieten, and J. Poisquet. 2012. "In-depth joint supply chain learning: towards a framework." Supply Chain Management: an International Journal 17 (6): 627-637.

- Lucio-Arias, D., and L. Leydesdorff. 2008. "Main path analysis and path-dependent transitions in HistCite based histograms." Journal of the American Society for Information Science and Technology. 59 (12): 1948-62.

- Lutz, H., and L. Birou. 2013. "Logistics education: a look at the current state of the art and science.” Supply Chain Management: An International Journal 18 (4): 455 467. 
- Mangan, J., and M. Christopher. 2005. "Management development and the supply chain manager of the future." The International Journal of Logistics Management. 16 (2): 178-191.

- Menon, S.T. 2012. "Human resource practices, supply chain performance, and wellbeing." International Journal of Manpower 33 (7): 769-785.

- Murphy, P. R., and R. F. Poist. 1994. "Educational strategies for succeeding in logistics: a comparative analysis." Transportation Journal 34 (3): 36-48.

- Murphy, P. R., and R. F. Poist. 2007. "Skill requirements of senior-level logisticians: a longitudinal assessment." Supply Chain Management: An International Journal 12 (6): 423-431.

- Onar, S.C., E. Aktas, Y. I. Topcu, and D. Doran. 2013. "An analysis of supply chain related graduate programmes in Europe." Supply Chain Management: an International Journal 18 (4): 398-412.

- Perera, S., S. O. Babatunde, L. Zhou, J. Pearson, and D. Ekundayo. 2016. “Competency mapping framework for regulating professionally oriented degree programmes in Higher Education." Studies in Higher Education DOI: $10.1080 / 03075079.2016 .1143926$

- Popa, V., D. Tanasescu, and I. Radulescu. 2009. "Project for human resources allignment at big challanges in SCM (supply chain management)." Metalurgia International 14: 152-158.

- Rahman, S., and N. Qing. 2014. "Graduate students' perceptions of supply chain skills for supply chain managers." Benchmarking: An International Journal 21 (2 ): 276 299.

- Schoenherr, T., and C. Speier-Pero. 2015. "Data Science, Predictive Analytics, and Big Data in Supply Chain Management: Current State and Future Potential." Journal of Business Logistics. 36 (1): 120-132.

- Sci2. 2009. "Science of Science (Sci2) Tool." Indiana University and SciTech Strategies. Available at: https://sci2.cns.iu.edu

- Sedelnikova, I., and N. Emelyanova. 2014. "Business Education: Developing Professional Competences through Gaming." Proceedings of the 2014 International Conference on Education Reform and Modern Management 1:298-302. 
- Shema, H. 2013. "What's wrong with citation analysis?" Available at: http://blogs.scientificamerican.com/information-culture/2013/01/01/whats-wrongwith-citation-analysis/

- Sodhi, M. S., B. G. Son, and C. S. Tang. 2008. "ASP, The Art and Science of Practice: What Employers Demand from Applicants for MBA-Level Supply Chain Jobs and the Coverage of Supply Chain Topics in MBA Courses." Interfaces 38 (6): 469-484.

- Sohal, A. S. 2013. "Developing competencies of supply chain professionals in Australia: collaboration between businesses, universities and industry associations." Supply Chain Management: An International Journal 18 (4): 429 - 439.

- Soshko, O., Y. Merkuryev, G. Merkuryeva, and J. Bikovska. 2005. "Development of active training and educational methods in logistics." Annual Proceedings of Vidzeme University College "ICTE in Regional Development" 1: 62-66.

- Thai, V. V., and G. T. Yeo. 2015. "Perceived competencies required for container shipping logisticians in Singapore and South Korea." International Journal of Logistics Management 26 (2): 334-355.

- Van Eck, N. J., and L. Waltman. 2013. VoSviewer. Centre for Science and Technology Studies, Leiden University. The Netherlands. Available at: http://www.vosviewer.com

- Van Eck, N. J., and L. Waltman. 2009. "How to normalize cooccurrence data? An analysis of some well-known similarity measures." Journal of the American Society for Information Science and Technology 60 (8): 1635-1651.

- Waller, M. A., and S. E. Fawcett. 2013. "Data Science, Predictive Analytics, and Big Data: A Revolution That Will Transform Supply Chain Design and Management." Journal of Business Logistics 34 (2): 77-84.

- Wang, Y., and A. J. Bowers. 2016. "Mapping the Field of Educational Administration Research: A Journal Citation Network Analysis of the Discipline." Journal of Educational Administration 54 (3): 242-269.

- Web of Science. 2014. Quick Reference Guide. Available at: http://wokinfo.com/media/pdf/wos_core_collection_qrc.pdf

- Wong, C. Y., D. B. Grant, B. Allan, and I. Jasiuvian. 2014. "Logistics and supply chain education and jobs: a study of UK markets." The International Journal of Logistics Management 25 (3): 537 - 552. 
- Wu, Y. C. J., S. K. Huang, M. Goh, and Y. J. Hsieh. 2013. "Global logistics management curriculum: perspective from practitioners in Taiwan." Supply Chain Management: an International Journal 18 (4): 376-388. 
Table 1 . Most frequently cited ten articles

\begin{tabular}{|c|c|c|c|}
\hline Rank & Author / Title / Journal / Date & LCS & GCS \\
\hline 1 & $\begin{array}{l}\text { Gravier MJ, Farris MT } \\
\text { An analysis of logistics pedagogical literature Past and future trends in curriculum, content, } \\
\text { and pedagogy } \\
\text { INTERNATIONAL JOURNAL OF LOGISTICS MANAGEMENT. } 2008 ; 19 \text { (2): 233-253 }\end{array}$ & 7 & 15 \\
\hline 2 & $\begin{array}{l}\text { Murphy P, Poist RF } \\
\text { Skill requirements of senior-level logisticians: a longitudinal assessment } \\
\text { SUPPLY CHAIN MANAGEMENT-AN INTERNATIONAL JOURNAL. 2007; } 12 \text { (6): } \\
\text { 423-431 }\end{array}$ & 4 & 16 \\
\hline 3 & $\begin{array}{l}\text { Sodhi MS, Son BG, Tang CS } \\
\text { ASP, The Art and Science of Practice: What Employers Demand from Applicants for MBA- } \\
\text { Level Supply Chain Jobs and the Coverage of Supply Chain Topics in MBA Courses } \\
\text { INTERFACES. } 2008 \text { NOV-DEC; } 38 \text { (6): } 469-484\end{array}$ & 2 & 10 \\
\hline 4 & $\begin{array}{l}\text { Lutz H, Birou L } \\
\text { Logistics education: a look at the current state of the art and science } \\
\text { SUPPLY CHAIN MANAGEMENT-AN INTERNATIONAL JOURNAL. 2013; } 18 \text { (4): } \\
455-467\end{array}$ & 2 & 1 \\
\hline 5 & $\begin{array}{l}\text { Agarwal R, Selen W } \\
\text { Dynamic Capability Building in Service Value Networks for Achieving Service Innovation } \\
\text { DECISION SCIENCES. } 2009 \text { AUG; } 40 \text { (3): 431-475 }\end{array}$ & 1 & 49 \\
\hline 6 & $\begin{array}{l}\text { Lambrechts F, Taillieu T, Grieten S, Poisquet J } \\
\text { In-depth joint supply chain learning: towards a framework } \\
\text { SUPPLY CHAIN MANAGEMENT-AN INTERNATIONAL JOURNAL. 2012; } 17 \text { (6): } \\
627-637\end{array}$ & 1 & 2 \\
\hline 7 & $\begin{array}{l}\text { Kovacs G, Tatham P, Larson PD } \\
\text { What Skills Are Needed to be a Humanitarian Logistician? } \\
\text { JOURNAL OF BUSINESS LOGISTICS. } 2012 \text { SEP; } 33 \text { (3): 245-258 }\end{array}$ & 1 & 8 \\
\hline 8 & $\begin{array}{l}\text { Aldous DE } \\
\text { Postharvest Horticulture Education and Training Opportunities in the Asia-Pacific Region } \\
\text { II ASIA PACIFIC SYMPOSIUM ON POSTHARVEST RESEARCH EDUCATION AND } \\
\text { EXTENSION (APS2012). 2013; 1011: 25-33 }\end{array}$ & 1 & 1 \\
\hline 9 & $\begin{array}{l}\text { Wu YCJ, Huang SK, Goh M, Hsieh YJ } \\
\text { Global logistics management curriculum: perspective from practitioners in Taiwan } \\
\text { SUPPLY CHAIN MANAGEMENT-AN INTERNATIONAL JOURNAL. 2013; } 18 \text { (4): } \\
\text { 376-388 }\end{array}$ & 1 & 1 \\
\hline 10 & $\begin{array}{l}\text { Onar SC, Aktas E, Topcu YI, Doran D } \\
\text { An analysis of supply chain related graduate programmes in Europe } \\
\text { SUPPLY CHAIN MANAGEMENT-AN INTERNATIONAL JOURNAL. 2013; } 18 \text { (4): } \\
398-412\end{array}$ & 1 & 1 \\
\hline
\end{tabular}


Table 2. Countries with the highest number of citation network articles

\begin{tabular}{rlr}
\hline$\#$ & \multicolumn{1}{c}{ Country } & Recs \\
\hline $\mathbf{1}$ & USA & 15 \\
$\mathbf{2}$ & UK & 8 \\
$\mathbf{3}$ & Australia & 7 \\
$\mathbf{4}$ & Peoples R China & 4 \\
$\mathbf{5}$ & Finland & 3 \\
$\mathbf{6}$ & Canada & 2 \\
$\mathbf{7}$ & Germany & 2 \\
$\mathbf{8}$ & India & 2 \\
$\mathbf{9}$ & Netherlands & 2 \\
$\mathbf{1 0}$ & Singapore & 2 \\
\hline
\end{tabular}




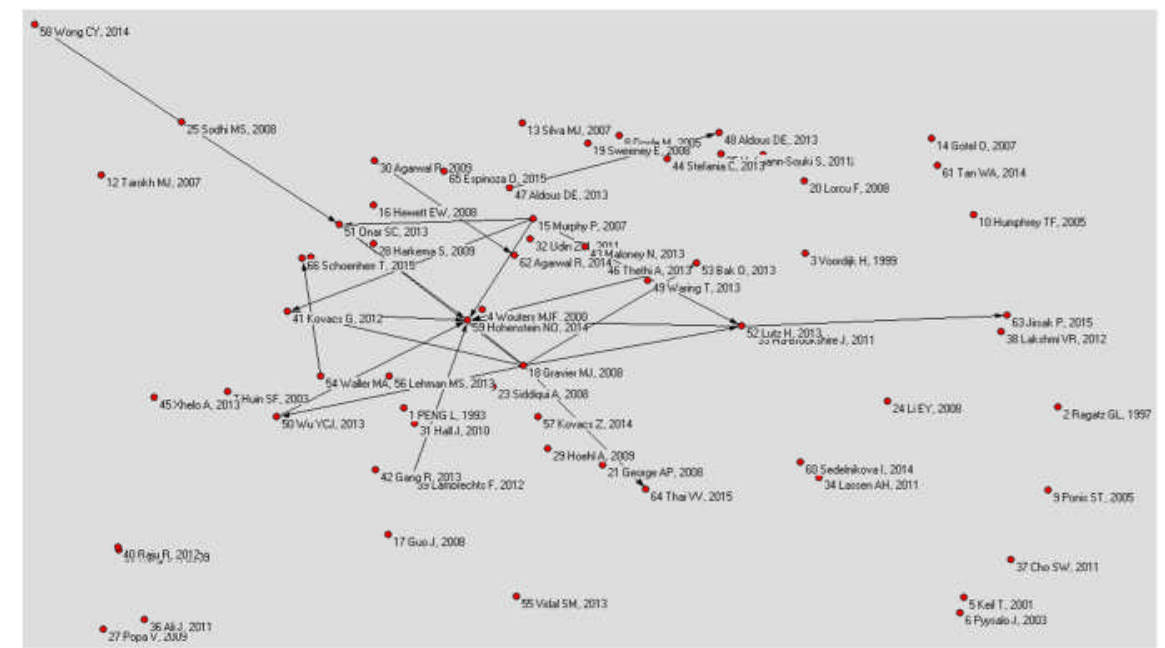

Figure 1. Citation Network (66 nodes)

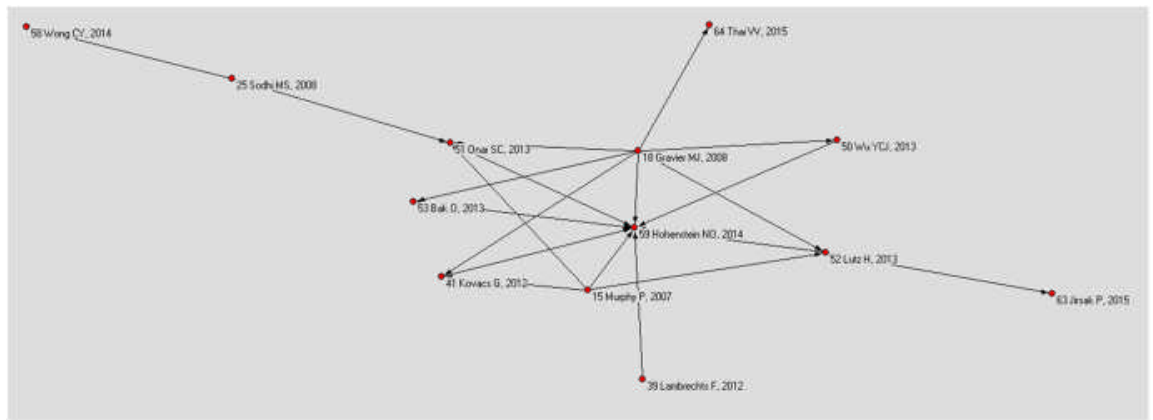

Figure 2. Main Connected Component (13 nodes)

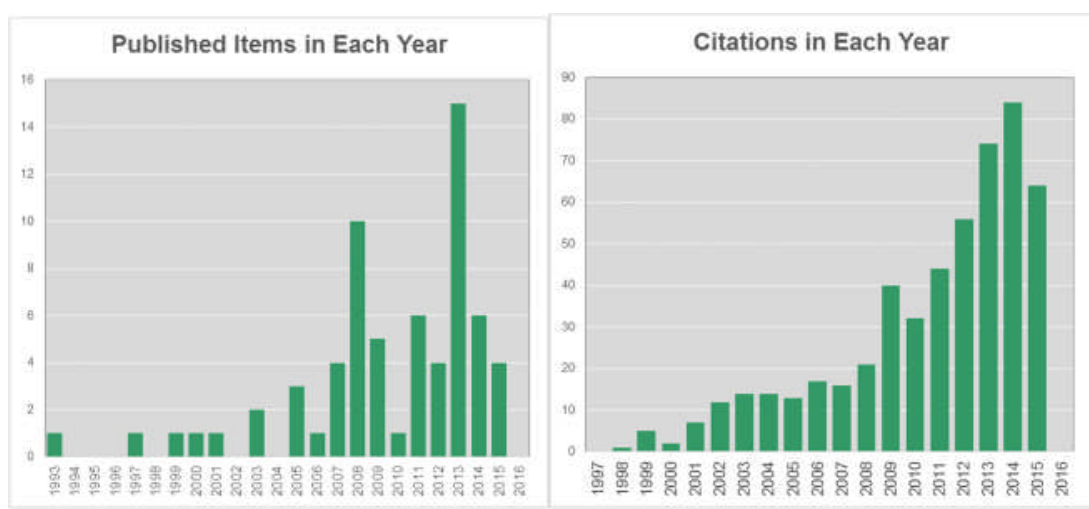

Figure $3 \mathrm{a} / \mathrm{b}$. Distribution of published scientific articles and citations 


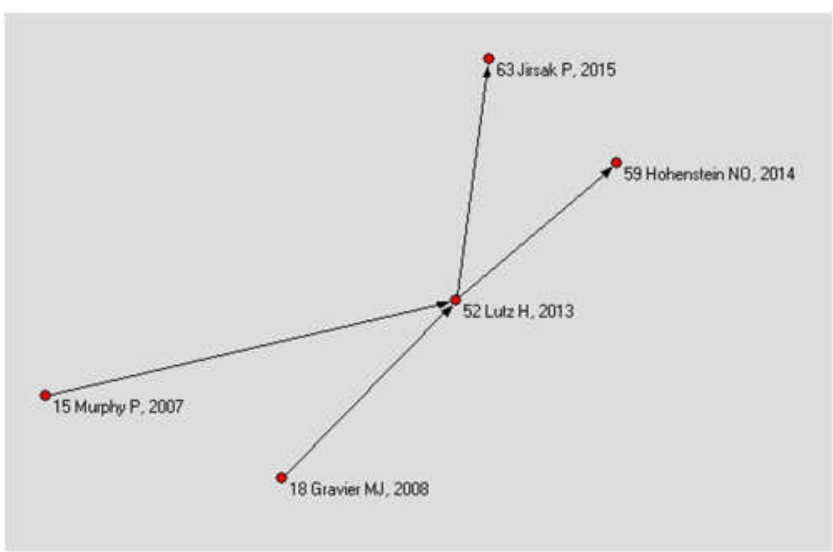

Figure 4. Main Path Component

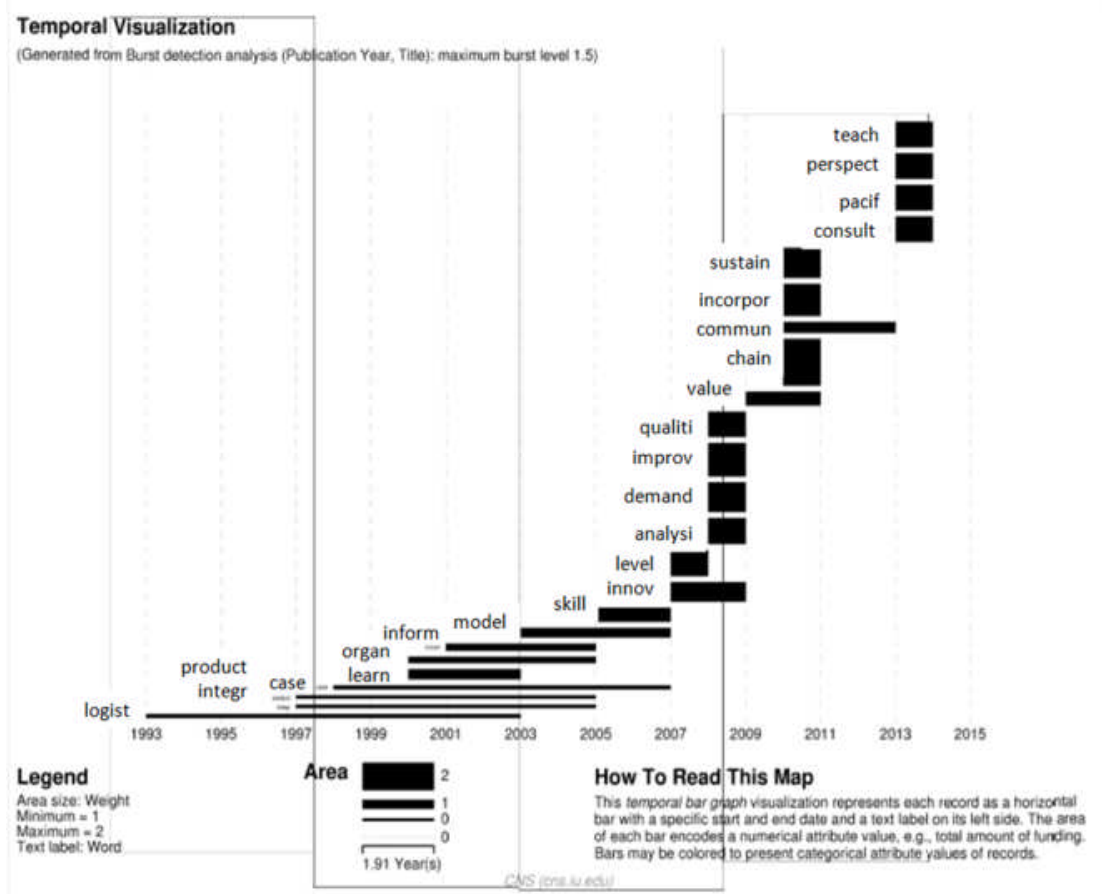

Figure 5. Burst detection of title words 


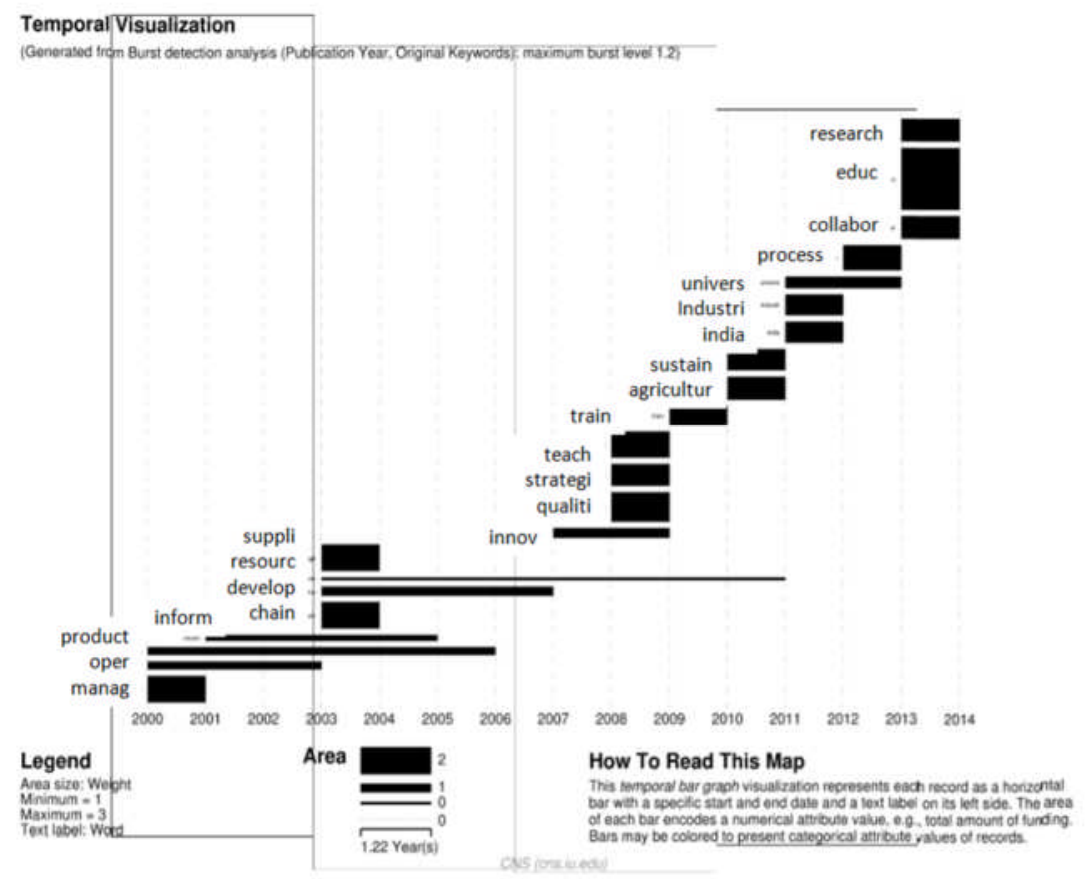

Figure 6. Burst detection of original keywords

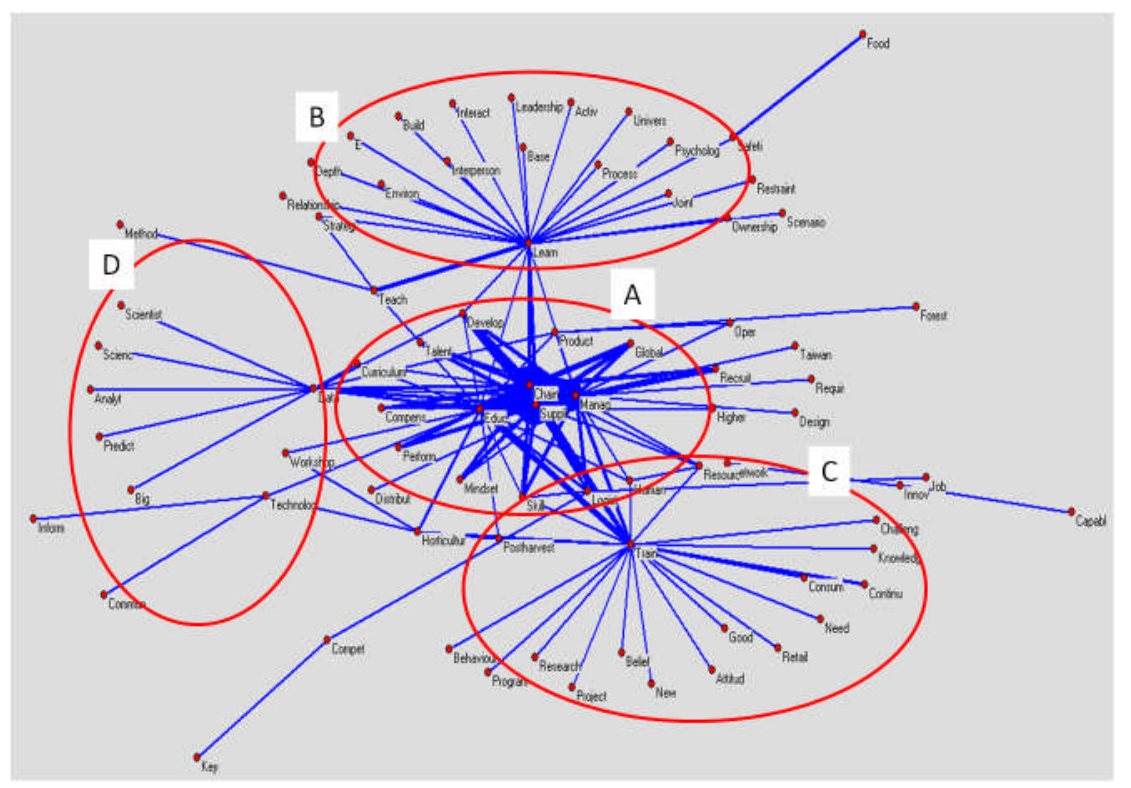

Figure 7. Original keywords network (size of arcs proportional to their weight) 


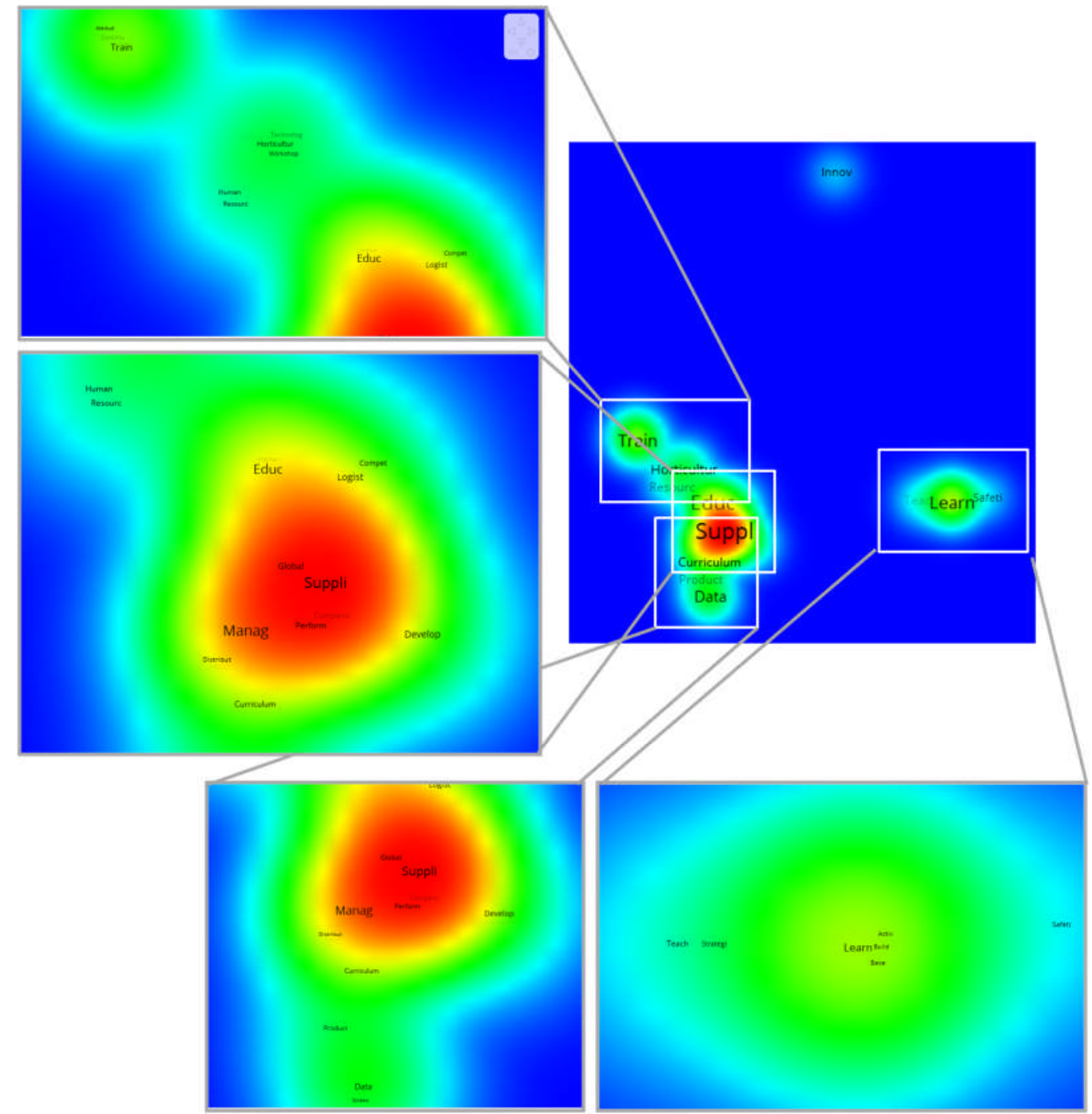

Figure 8. Authors' keywords VOS mapping 